Instituto Internacional de Investigación y Desarrollo Tecnológico Educativo INDTEC, C.A.

DOI: https://doi.org/10.29394/Scientific.issn.2542-2987.2021.6.20.5.92-112

OAl-PMH: http://www.indteca.com/ojs/index.php/Revista Scientific/oai

Artículo Original / Original Article

\title{
Competencias y recursos digitales para la enseñanza aprendizaje en educación básica superior
}

Autores: Jenny Patricia León Amendaño Universidad Católica de Cuenca, UCACUE jennypatricia.leon@est.ucacue.edu.ec Cuenca, Ecuador https://orcid.org/0000-0002-1290-6569

Pablo Fernando Cisneros Quintanilla Universidad Católica de Cuenca, UCACUE pcisneros@ucacue.edu.ec Cuenca, Ecuador https://orcid.org/0000-0002-5722-8001

\section{Resumen}

El presente artículo tiene por objetivo identificar recursos digitales para el desarrollo de las siguientes competencias digitales: a). informatización y alfabetización informacional; b). comunicación y colaboración; c). resolución de problemas y pensamiento crítico; y d). seguridad. La obtención de estas competencias digitales, permitirá una correcta gestión de la información y el uso crítico y seguro de recursos digitales, para así contribuir a la calidad de la educación de la Unidad Educativa Particular "Santa Mariana de Jesús", Cuenca, Ecuador, donde se ha detectado una insuficiencia en el desarrollo de competencias digitales. En este artículo con enfoque cuantitativo, forma parte del trabajo de grado de la maestría en Pedagogía de la Universidad Católica de Cuenca, se aplicó una encuesta a 90 estudiantes y 10 docentes, que proporcionó información sobre el nivel de conocimientos de recursos y competencias digitales. Los datos obtenidos muestran interesantes resultados que denotan una diferencia significativa en el uso de recursos digitales entre estudiantes y docentes; además, los docentes consideran de mucha importancia la necesidad de desarrollar competencias digitales. En conclusión, existe la necesidad de integrar recursos digitales y capacitar a docentes y estudiantes sobre el uso eficaz de recursos para el desarrollo de competencias digitales.

Palabras clave: competencias digitales; recursos educativos digitales; aprendizaje; enseñanza.

Código de clasificación internacional: 5803.02 - Preparación de profesores.

\footnotetext{
Cómo citar este artículo:

León, J., \& Cisneros, P. (2021). Competencias y recursos digitales para la enseñanza aprendizaje en educación básica superior. Revista Scientific, 6(20), 92-112, e-ISSN: 2542-2987. Recuperado de: https://doi.org/10.29394/Scientific.issn.2542-2987.2021.6.20.5.92-112
}

Fecha de Recepción:

07-01-2021
Fecha de Aceptación:

20-03-2021
Fecha de Publicación: 05-05-2021 
OAl-PMH: http://www.indteca.com/ojs/index.php/Revista Scientific/oai

Artículo Original / Original Article

\title{
Competences and digital resources for teaching-learning in basic higher education
}

\begin{abstract}
This article aims to identify digital resources for the development of the following digital skills: a). computerization and information literacy; b). communication and collaboration; c). problem solving and critical thinking; and d). safety. Obtaining these digital competences will allow a correct management of information and the critical and safe use of digital resources, in order to contribute to the quality of education of the Private Educational Unit "Saint Mariana of Jesus", Cuenca, Ecuador, where an insufficiency in the development of digital competences has been detected. In this article with a quantitative approach, it is part of the degree work of the master's degree in Pedagogy at the Catholic University of Cuenca, a survey was applied to 90 students and 10 teachers, which provided information on the level of knowledge of digital resources and skills. The data obtained show interesting results that denote a significant difference in the use of digital resources between students and teachers; Furthermore, teachers consider the need to develop digital skills to be very important. In conclusion, there is a need to integrate digital resources and train teachers and students on the effective use of resources for the development of digital skills.
\end{abstract} teaching.

Keywords: digital skills; digital educational resources; learning; International classification code: 5803.02 - Training of teachers.

\footnotetext{
How to cite this article:

León, J., \& Cisneros, P. (2021). Competences and digital resources for teaching-learning in basic higher education. Revista Scientific, 6(20), 92-112, e-ISSN: 2542-2987. Recovered from: https://doi.org/10.29394/Scientific.issn.2542-2987.2021.6.20.5.92-112
}

Date Received: 07-01-2021
Date Acceptance: 20-03-2021
Date Publication: 05-05-2021 


\section{Introducción}

La necesidad de incorporar las competencias digitales en la formación profesional, obliga a los usuarios a ser capaces de utilizar las Tecnologías de Información y Comunicación (TIC) de forma segura y eficaz. En el campo educativo, los docentes y estudiantes son usuarios activos de las TIC, por tanto, estos no deben ignorar el desarrollo de las competencias digitales en el proceso educativo, ya que dichas competencias digitales permitirán un desenvolvimiento eficaz con las TIC; y así convertir a las TIC en un medio eficiente dentro del campo educativo.

En este sentido, Pozos y Tejada (2018): proponen que la adquisición de competencias digitales se ha vuelto necesaria, ante la importancia que han adquirido las TIC en los actuales y futuros escenarios en los que se desenvuelve la ciudadanía. Esos escenarios colocan a la población en general y a los maestros en particular, ante nuevos roles que exigen no solo las tradicionales competencias docentes, sino el dominio de competencias digitales que no pueden ser pensadas únicamente en el manejo instrumental de las TIC, sino en la gestión integral de la información.

Es por tanto, muy importante indicar la conceptualización de competencias digitales de García-Quismondo y Cruz-Palacios (2018): como aquellas herramientas educativas que permiten a los ciudadanos participar en la empleabilidad, la economía, la política, el ocio y la cultura del siglo XXI.

En igual sentido, la Organización de las Naciones Unidas para la Educación, la Ciencia y la Cultura (UNESCO, 2019a): plantea que para el desarrollo de competencias digitales en los estudiantes, es necesario que los profesores adquieran competencias digitales con el propósito de mejorar el uso de dispositivos, herramientas y aplicaciones digitales, para acceder y gestionar la información, pero además para utilizar adecuadamente dichos recursos digitales que le permitan crear, compartir y ejecutar tareas interactivas tanto individuales como colaborativas. 
Complementa la misma Organización de las Naciones Unidas para la Educación, la Ciencia y la Cultura (UNESCO, 2019b): que estas competencias en docentes y estudiantes, fomentan la creación e intercambio de contenidos digitales, la comunicación y la colaboración, elementos fundamentales para proponer soluciones a los problemas del entorno social y alcanzar un desarrollo eficaz, contribuyendo así a la calidad de la enseñanza.

Es así como, desarrollar competencias digitales durante el proceso de enseñanza aprendizaje, requiere una correcta integración de recursos digitales en las clases presenciales o virtuales, para lo que los profesores deben estar formados e informados en aspectos relativos a lo digital, especialmente por la transición actual de clases presenciales a virtuales.

En párrafos anteriores se han analizado los conceptos de competencias digitales, desde el punto de vista de diferentes autores, ahora es importante analizar la conceptualización según el autor Montaño (2016), que se refiere al concepto recurso digital en los siguientes términos:

Elementos mediadores útiles para el desarrollo del proceso de enseñanza-aprendizaje, como el pizarrón, cartel, papelógrafo, proyector y herramientas digitales de audio, voz, procesadores de datos y páginas web; medios actuales que facilitan mejoras en la función del docente y estudiante, fomentando la motivación e interés de ambos entes y al mismo tiempo haciendo de la educación más dinámica e interactiva (pág. 15).

En conclusión, un recurso digital es aquel elemento activo de gran utilidad educativa en formato digital, el cual se puede visualizar y almacenar en un dispositivo electrónico o también se lo puede consultar directamente en el Internet. Sin embargo, existe la problemática de una insuficiencia en el desarrollo de competencias digitales para el aprendizaje en los estudiantes, puesto que, los docentes tampoco cuentan con la capacitación para aprovechar distintos recursos digitales.

Es así que, después de haber analizado los conceptos de competencias 
y de recursos digitales, en los párrafos siguientes, se presenta una compilación de diferentes investigaciones relacionadas con las competencias y los recursos digitales, las cuales se dividen en cuatro contextos: mundial, latinoamericano, ecuatoriano y cantonal.

En el primer contexto mundial, en torno al uso de recursos digitales, se examinó la investigación descriptiva de Venegas (2017a): quién tuvo como objetivo general evaluar un programa de enseñanza de las matemáticas en 46 alumnos de sexto de primaria de Salamanca, en base a una selección de recursos digitales de calidad, con el análisis de las implicaciones en el aprendizaje, motivación y satisfacción de los estudiantes, donde la autora en su tesis seleccionó 37 de 161 recursos digitales, afirmando que los recursos fueron elegidos por la cantidad en contenido y/o los ejercicios propuestos y por su valor didáctico.

Entre las conclusiones obtenidas en la tesis doctoral antes mencionada, cuando la autora analiza el aspecto motivacional al utilizar recursos TIC, tales como ordenadores, pizarras digitales interactivas (PDI), internet, entre otros. En ese marco, Venegas (2017b), destaca que:

Los alumnos manifiestan que los recursos TIC (ordenador, PDI, Internet) les motiva para aprender en matemáticas. En los resultados casi el $100 \%$ de la muestra manifiesta que le gustaría seguir aprendiendo en clase de matemáticas con el ordenador y la PDI (97,8\%) (pág. 267).

En el argumento latinoamericano para referirse al consumo informativo, se analizó una investigación con enfoque cuantitativo, propuesta por RomeroRodríguez y Aguaded (2016a): como objetivo para estudiar a profundidad los hábitos de los estudiantes de grado de comunicación en Colombia, Perú y Venezuela, en particular sobre la infoxicación y sobresaturación informativa, para lo cual, los autores aplicaron un cuestionario digital.

Conforme a lo anterior, Romero-Rodríguez y Aguaded (2016b): 
presentaron como conclusiones en su trabajo investigativo que existe la necesidad de una formación temprana de competencias digitales, informacionales y mediáticas como requisito indispensable para el acceso a una carrera profesional, donde se debe inculcar la responsabilidad formativa sobre el uso de los medios digitales y las redes sociales.

Por otro lado, en lo que respecta al ámbito nacional, se seleccionó una investigación cuantitativa sobre interaprendizaje como lo demuestran Vásquez-Aguilar, Dominguez y Mata-Muñoz (2017): quienes tuvieron como propósito optimizar el rendimiento académico y el desarrollo cognitivo de 30 estudiantes de la asignatura de Relaciones Laborales de la Carrera Ingeniería Industrial de la Universidad de Guayaquil, para que los estudiantes construyan metodologías, competencias y mentalidades innovadoras, emprendedoras con una visión de desempeño eficiente y eficaz para el cumplimiento de sus objetivos.

En lo que corresponde al Cantón Cuenca, se utilizó un estudio de caso único descriptivo sobre las competencias digitales de Flores y Garrido (2019a): quienes presentaron como objetivo "[...] analizar y reflexionar sobre las fortalezas, las debilidades, las limitaciones, la postura frente al uso de las TIC y la integración en los procesos formativos [...]" (pág. 44).

De manera que, Flores y Garrido (2019b): para su investigación, tomaron como muestra 82 docentes de carreras de educación básica, inicial, especial, interculturalidad bilingüe y profesionalización durante el año lectivo 2018-2019, a quienes aplicaron un cuestionario semiestructurado de 10 preguntas.

Con la aplicación del cuestionario, es importante señalar la conclusión a la que llegaron Flores y Garrido (2019c): sobre la "[...] necesidad emergente de capacitar a los docentes universitarios en el uso de las tecnologías, pero no solamente en la adquisición de competencias básicas, será necesario preparar a los docentes en una verdadera integración de las TIC [...]" (pág. 
56); donde el desarrollo de competencias digitales trascienda el aspecto meramente instrumental y que se oriente al desempeño profesional.

\subsection{Competencias digitales en estudiantes}

La sociedad de la información, la cual está caracterizada por la informatización y la mediatización, permite a los estudiantes acceder a la información instantáneamente y en grandes cantidades, sin embargo, los alumnos deben desarrollar las competencias digitales, para convertir la acertada información en aprendizajes significativos. En los párrafos siguientes se analizarán las siguientes competencias digitales: a). informatización y alfabetización informacional; b). comunicación y colaboración; c). resolución de problemas y pensamiento crítico; y d). seguridad. De igual manera se identificaron algunos recursos digitales para el desarrollo de esas competencias digitales.

\subsubsection{Competencia digital: informatización y alfabetización informacional}

Para comprender la capacidad de informatización y alfabetización informacional es importante recalcar la opinión de Caccuri (2018a): en cuanto a que "esta competencia se promueve estimulando a los estudiantes a buscar resultados concretos y discutir sobre la información hallada" (pág. 10).

En concordancia con la autora, el papel del estudiante debe ir más allá de ser un lector de información, pues se requiere que sea capaz de analizar, sintetizar, evaluar y difundir la información, con el empleo de buscadores de información fidedigna y acorde a la edad del usuario, por lo cual, los docentes y estudiantes conjuntamente con los padres de familia deben hacer uso de recursos digitales de información que brinden seguridad para los estudiantes, en aspectos relativos tanto al origen de la información como a la influencia que esta pudiera ejercer en las personas que la utilizan. 


\subsubsection{Recursos digitales para favorecer la competencia digital de informatización y alfabetización informacional}

El sitio web denominado Bunis, es un espacio virtual de navegación segura que bloquea contenido inapropiado, donde los profesores y representantes podrán crearse una cuenta en el sitio web de forma gratuita para controlar las búsquedas realizadas por los estudiantes, informarse sobre los términos de búsqueda de los alumnos y verificar el uso en varios dispositivos que maniobra el estudiante.

La página web, Buscador para niños, es un sitio seguro de búsqueda para menores, que brinda un sistema de filtro de contenidos inapropiados para niños, además de dispones de distintas actividades para que desarrollen los estudiantes, este buscador permite que los estudiantes retroalimenten los aprendizajes con diversas e interactivas actividades en línea.

Por consiguiente, la utilización de estos navegadores seguros debe trabajarse colaborativamente entre profesores, estudiantes y padres de familia, pues si en el entorno de enseñanza aprendizaje se utiliza uno o varios de estos navegadores, se debe promover el uso de estos en el entorno familiar de los estudiantes para facilitar la adquisición de aprendizajes significativos.

\subsubsection{Competencia digital: comunicación y colaboración}

En esa misma línea, Caccuri (2018b): define a la competencia digital de comunicación y colaboración como la "habilidad para comunicarse en entornos digitales, compartir recursos por medio de herramientas en red, conectar con otros y colaborar mediante herramientas digitales, interaccionar y participar en comunidades y redes, con conciencia intercultural" (pág. 12).

En tal sentido, es factible que los profesores utilicen diferentes metodologías y compartan con sus alumnos diversos recursos digitales para aprovechar al máximo la tecnología y así evitar que las aulas virtuales se conviertan en espacios a los que se han trasladado las viejas prácticas de la 
educación tradicional, pero con la única novedad de la incorporación de las TIC.

\subsubsection{Recursos digitales para favorecer la competencia digital Comunicación y colaboración}

La plataforma educativa Edmodo, es un recurso digital, que a juzgar por Díaz (2017):

Permite establecer un espacio virtual de comunicación con los estudiantes y docentes, en el que se pueden hacer comentarios y aportes de las actividades realizadas, adjuntar archivos y enlaces, establecer un calendario de trabajo, así como de actividades, evaluaciones y gestionarlas (pág. 10).

En función de lo anterior, al ser Edmodo una plataforma social educativa, permite la conexión y comunicación entre profesores, estudiantes y padres de familia donde cada usuario podrá crearse una cuenta gratis para interactuar, colaborar y comunicarse entre sí de manera privada y podría decirse exclusivamente para el aprendizaje.

La herramienta digital MindMeister, tal como la conceptualiza Caccuri (2018c): es una "aplicación para elaborar mapas conceptuales en línea y de forma colaborativa. Permite insertar multimedia, gestionar y asignar tareas y convertirlos en una presentación o en un documento imprimible" (pág. 14).

Esta aplicación tiene como fin el trabajo colaborativo, pues además de generar mapas conceptuales permite el desarrollo de lluvias de ideas en tiempo real desde cualquier sitio o dispositivo, aquí es clave la orientación del docente para perfeccionar la capacidad de síntesis del estudiante, además de promover la asistencia entre compañeros utilizando lluvias de ideas para la construcción de mapas conceptuales.

La plataforma de colaboración Stormboard, permite desarrollar lluvias de ideas, de este modo, Caccuri (2018d): dice que "permite compartir una 
pizarra para que todo el grupo añada sus aportes. Cada idea agregada incluye un hilo de conversación de su creador" (pág. 14). Además, esta plataforma permite trabajar remotamente desde una diversidad de equipos, lo que propicia el desarrollo de debates que promuevan el diálogo donde cada aportación comentario o propuesta, permitiría a los estudiantes convertirse en los protagonistas de su aprendizaje, enriqueciendo así también el conocimiento al trabajar colaborativamente.

\subsubsection{Competencia digital: resolución de problemas y pensamiento crítico}

La resolución de problemas es una competencia que se puede lograr a través del pensamiento crítico, lo que aporta significativamente al contexto educativo, pues los docentes y estudiantes serían capaces de incorporar habilidades, actitudes, reflexiones para la construcción de aprendizajes o conocimientos significativos. En este orden de ideas, Caccuri (2018e), indica que la resolución de problemas:

Implica identificar necesidades de uso de recursos digitales, tomar decisiones informadas sobre las herramientas digitales más apropiadas según el propósito o la necesidad, resolver problemas conceptuales a través de medios digitales, usar las tecnologías de forma creativa, resolver problemas técnicos, actualizar su propia competencia y la de otros (pág. 21).

En concordancia con el párrafo anterior, es importante recalcar que los individuos están expuestos a situaciones que implican la capacidad de afrontar y resolver situaciones de conflicto, para lo cual aquellos individuos en especial los estudiantes, deben desarrollar las capacidades para la resolución de problemas, donde el pensamiento crítico sea auto dirigido, auto disciplinado y auto corregido.

De esta forma, Valencia-Molina, Serna-Collazos, Ochoa-Angrino, Caicedo-Tamayo, Montes-González y Chávez-Vescance (2016): se refieren al 
pensamiento crítico como "las habilidades para utilizar diferentes tipos de razonamiento, hacer juicios y tomar decisiones apoyándose en la evaluación en evidencia y argumentos y la resolución de problemas" (pág. 14). Por lo tanto, el pensamiento crítico como competencia digital promueve en el estudiante el análisis y la evaluación de la información que recibe de diferentes canales, sean virtuales o físicos, para así obtener conclusiones de las fuentes de información y determinar cuál de ellas es la idónea para construir los aprendizajes.

Además, de lo anterior se desprende el rol del docente, el cual debe fomentar en los estudiantes el cuestionamiento sobre lo que leen, los autores y sus ideas, pues, es importante no llenarse de fuentes de información, ya que lo significativo es analizar las fuentes y elegir la idónea para adquirir aprendizajes.

\subsubsection{Recursos digitales para favorecer la competencia resolución de problemas y pensamiento crítico}

El portal educativo Eduteka, es un recurso digital que pone a disposición de docentes y personal de instituciones educativas, documentos, artículos de divulgación y difusión de manera gratuita; módulos temáticos para la autoformación del docente; proyectos de clase listos para ser utilizados; recursos digitales; herramientas TIC; y, un recurso para construir proyectos de clase personalizados. Este sitio virtual, es ofrecido por la Universidad Icesi (originalmente las siglas de Instituto Colombiano de Estudios Superiores de Incolda) que busca aportar para la mejora continua de los ambientes de enseñanza y aprendizaje, sean estos presenciales o virtuales.

La comunidad virtual titulada REDuteka, es un espacio web en el que participan los usuarios registrados en el portal educativo Eduteka, que permite entre otras cosas propias de las redes sociales, la generación de documentos, recursos y herramientas digitales de uso educativo, logrados mediante el 
trabajo colaborativo de los miembros de esta comunidad.

\subsubsection{Competencia digital: seguridad}

Dentro del campo educativo, para describir a la competencia seguridad, según Caccuri (2018f): "esta competencia abarca la protección de información y datos personales, la protección de la identidad digital, las medidas de seguridad y el uso responsable y seguro de internet" (pág. 18).

En concordancia con la autora mencionada, es importante considerar la especial vulnerabilidad en la que, por su desarrollo evolutivo, se encuentran los estudiantes de diez a trece años, por tal razón, las buenas prácticas para la seguridad de cualquier individuo y de manera particular de los estudiantes en las edades mencionadas, implican que se prevea la seguridad de su identidad digital y privacidad de estos usuarios que emplean las TIC.

Por lo que es prioritario que además de los padres de familia, los profesores guíen a los estudiantes con actividades que fomenten su seguridad para comprender, analizar los términos y condiciones de uso de sitios web, socializar consejos y recomendaciones para la protección de datos personales, utilizando la ejemplificación de diferentes situaciones que afectan o comprometen los datos personales y comprender las consecuencias de poner a disposición de cualquier persona ese tipo de información.

\subsubsection{Recursos digitales para favorecer la competencia digital seguridad}

El portal web Segureskola, es un portal que ofrece un programa integral que coordina y capacita a colegios y familias para mejorar la ciberseguridad, de tal manera que la supervisión digital sea efectiva, situación que implica la necesidad de incorporar costos a los programas según la necesidad de la institución educativa. Sin embargo, este sitio ofrece la suscripción a su revista digital que envía mensual y gratuitamente artículos, además ofrece un canal 
de educación digital en YouTube, que permite la adquisición de aprendizajes en materia de ciberseguridad.

En conclusión, las competencias digitales que se deben adquirir en el proceso de enseñanza aprendizaje la básica superior son: a). informatización y alfabetización informacional; b). comunicación y colaboración; c). resolución de problemas y pensamiento crítico; y d). seguridad, las competencias digitales antes mencionadas permitirán el uso seguro, creativo y eficiente de la tecnología y recursos digitales.

Es por lo que el presente artículo tiene por objetivo identificar recursos digitales para el desarrollo de las competencias mencionadas. La obtención de estas aptitudes digitales, permitirá una correcta gestión de la información y el uso crítico y seguro de recursos digitales, para así contribuir a la calidad de la educación de la Unidad Educativa Particular "Santa Mariana de Jesús", Cuenca, Ecuador.

\section{Metodología}

Considerando la problemática planteada, en torno al uso de recursos digitales para favorecer el desarrollo de competencias digitales por parte de los docentes-estudiantes y, motivo del objetivo de la investigación, donde la variable dependiente es el desarrollo de competencias digitales y la variable independiente es el aprovechamiento de recursos digitales, se decidió trabajar con una metodología descriptiva con enfoque cuantitativo.

Es así que, en la investigación se empleó el método empírico de medición con la aplicación de una encuesta como técnica y un cuestionario como instrumento de investigación, puesto que es un formato simple que facilitó el análisis y redujo los costos de aplicación pues, por la pandemia que se vive, se aplicó la encuesta utilizando los formularios de Google de manera virtual a docentes y estudiantes de octavo grado.

Por lo tanto, el universo de estudio estuvo conformado por 329 
estudiantes y 19 docentes de octavo grado, pero se trabajó únicamente con una muestra no probabilística de 90 estudiantes y 10 docentes que corresponden al octavo grado de la institución: Santa Mariana de Jesús en el año lectivo 2020-2021 para la selección de la muestra, se ha empleado el método no probabilístico por juicio, pues esta selección contribuye y se adapta significativamente a la presente investigación descriptiva.

De los 90 estudiantes pertenecientes al octavo grado, paralelos A, B y $\mathrm{C}$, de la institución educativa, que participaron en el estudio, el $77.80 \%$ corresponde al género femenino y el $22.20 \%$ al género masculino. Sus edades oscilan entre los 11 y 13 años. Así también, de los 10 docentes, el 80\% corresponde al género femenino y el $20 \%$ corresponde al género masculino. Sus edades oscilan entre los 25 y 50 años.

\section{Resultados}

Luego de la aplicación de la encuesta a docentes y estudiantes, se obtuvieron como resultados más relevantes los siguientes: en el gráfico 1, se puede observar que el $40 \%$ de docentes están totalmente de acuerdo en que los recursos digitales son útiles para desarrollar competencias digitales.

Gráfico 1. Postura del docente frente al uso de los recursos digitales para desarrollar competencias digitales.

Fuente: Los Autores (2021). 
Con los resultados obtenidos, es importante recalcar que la postura de los encuestados contribuiría significativamente al proceso de aprendizaje, ya que los docentes encuestados poseen una actitud reflexiva sobre la importancia de las competencias digitales.

Del total de los encuestados, el $90 \%$ de estudiantes consideran necesario desarrollar competencias digitales en su proceso de aprendizaje. Sin embargo, como se puede evidenciar en el gráfico 2 , solamente el $13 \%$ de estudiantes indican que poseen bastantes conocimientos sobre las competencias digitales, lo cual es insuficiente en una institución educativa.

Gráfico 2. Conocimiento de los estudiantes sobre las competencias digitales.

Fuente: Los autores (2021).

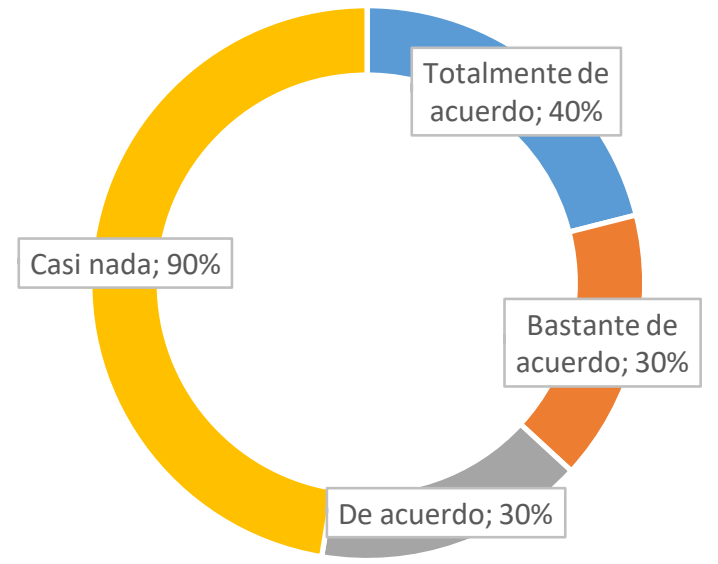

- Totalmente de acuerdo

- Bastante de acuerdo

- De acuerdo

- Casi nada

- Casi de acuerdo

En la tabla 1, se evidencia que existe una diferencia entre docentes y estudiantes en torno al uso de internet, correo electrónico, chat, plataformas para videoconferencia y paquete de office, entre las opciones que han sido elegidas mayoritariamente.

Los resultados obtenidos muestran una situación que no favorece el desarrollo de competencias digitales para adquirir conocimientos por parte de 
los estudiantes, pues necesitan de una práctica constante para lograr el uso eficaz de distintos recursos digitales que contribuyan significativamente a las competencias digitales, con una frecuencia de 10 horas semanales del $70 \%$ (docentes) y el $52.22 \%$ (estudiantes).

Tabla 1. Frecuencia de uso de recursos digitales: más de 10 horas semanales.

\begin{tabular}{|l|l|l|}
\hline Frecuencia de uso: más de 10 horas semanales & Docentes & Estudiantes \\
\hline Internet & $70 \%$ & $52.22 \%$ \\
\hline Correo Electrónico & $60 \%$ & $3.33 \%$ \\
\hline Chat & $60 \%$ & $26.67 \%$ \\
\hline Foros y blogs & $10 \%$ & $6.67 \%$ \\
\hline Herramientas de trabajo colaborativo & $10 \%$ & $17.78 \%$ \\
\hline Plataformas para videoconferencia & $70 \%$ & $45.56 \%$ \\
\hline Editores de imágenes & $20 \%$ & $10 \%$ \\
\hline Editores de videos & $20 \%$ & $10 \%$ \\
\hline Editores de audio & $20 \%$ & $5.56 \%$ \\
\hline Redes sociales & $40 \%$ & $25.56 \%$ \\
\hline Paquete de Office & $60 \%$ & $8.89 \%$ \\
\hline Video juegos & $10 \%$ & $21.11 \%$ \\
\hline
\end{tabular}

Fuente: Los autores (2021).

Con los datos obtenidos, se puede evidenciar que los docentes son quienes en su mayoría utilizan los recursos digitales para su desarrollo profesional, sin embargo, los estudiantes utilizan los recursos en menor medida, lo cual dificulta que desarrollen las competencias digitales para adquirir conocimientos, pues necesitan de una práctica constante para un uso eficaz de distintos recursos digitales que contribuyan significativamente a su proceso de aprendizaje.

\section{Conclusiones}

Los resultados de esta investigación de tipo descriptiva, han demostrado la necesidad de integrar distintos recursos digitales en el proceso de enseñanza aprendizaje, además de aquello, es imprescindible capacitar a los docentes y a su vez a los estudiantes sobre el uso de una variedad de 
distintos recursos para desarrollar competencias digitales.

En el mismo sentido, se evidencia que son los docentes los que tienen mayor práctica con distintos recursos digitales, sin embargo, deben propender a la innovación en su enseñanza con el uso de la tecnología que facilite el aprendizaje de los estudiantes en cualquier espacio y tiempo. Para esto, el estudiante deberá desarrollar como mínimo las siguientes competencias digitales: a). informatización y alfabetización informacional; b). comunicación y colaboración; c). resolución de problemas y pensamiento crítico; y d). seguridad.

Las competencias mencionadas anteriormente, promueven nuevas formas de enseñar y aprender con la integración eficaz de las TIC, donde se empleen las tecnologías para innovar en los procesos de enseñanza aprendizaje y así cumplir eficientemente con objetivos educativos planteados para procurar la mejora continua del proceso educativo.

Por lo mencionado, es de suma importancia trabajar en el desarrollo de competencias digitales desde temprana edad, en este caso, en el nivel básico, con los estudiantes de octavo grado, hasta alcanzar conocimientos, habilidades y destrezas que permitan utilizar eficientemente las tecnologías de la información y la comunicación como un medio puesto al servicio del proceso de enseñanza y aprendizaje.

\section{Referencias}

Caccuri, V. (2018a,b,c,d,e,f). Competencias Digitales para la Educación del Siglo XXI. e-book. Buenos Aires, Argentina: Autor.

Díaz, J. (2017). Edmodo como herramienta virtual de aprendizaje. Innova Research Journal, 2(10), 9-16, e-ISSN: 2477-9024. Recuperado de: https://doi.org/10.33890/innova.v2.n10.2017.259

Flores, D., \& Garrido, J. (2019). Competencias digitales para los nuevos escenarios de aprendizaje en el contexto universitario. Revista 
Scientific, 4(14), 44-61, e-ISSN: 2542-2987. Recuperado de:

https://doi.org/10.29394/Scientific.issn.2542-2987.2019.4.14.2.44-61

García-Quismondo, M., \& Cruz-Palacios, E. (2018). Gaming como

Instrumento Educativo para una Educación en Competencias

Digitales desde los Academic Skills Centres. Revista General de Información y Documentación, 28(2), 489-506, e-ISSN: 1132-1873.

Recuperado de: https://doi.org/10.5209/RGID.62836

Montaño, D. (2016). Evaluación de herramientas digitales para la gestión de portafolio educativo. Tesis de Maestría. Esmeraldas, Ecuador: Pontificia Universidad Católica del Ecuador- PUCESE. Recuperado de: https://repositorio.pucese.edu.ec/handle/123456789/601

Pozos, K., \& Tejada, J. (2018). Competencias Digitales en Docentes de Educación Superior: Niveles de Dominio y Necesidades Formativas. Revista Digital de Investigación en Docencia Universitaria, 12(2), 59-87, e-ISSN: 2223-2516. Recuperado de:

https://doi.org/10.19083/ridu.2018.712

Romero-Rodríguez, L., \& Aguaded, I. (2016). Consumo informativo y competencias digitales de estudiantes de periodismo de Colombia, Perú y Venezuela. Convergencia. Revista de Ciencias Sociales, 23(70), 35-57, e-ISSN: 1405-1435. Recuperado de:

https://www.redalyc.org/articulo.oa?id=10543305002

UNESCO (2019a,b). Marco de competencias de los docentes en materia de TIC UNESCO. ISBN: 978-92-3-300121-3. París, Francia: Organización de las Naciones Unidas para la Educación, la Ciencia y la Cultura.

Valencia-Molina, T., Serna-Collazos, A., Ochoa-Angrino, S., Caicedo-Tamayo, A., Montes-González, J., \& Chávez-Vescance, J. (2016). Competencias y estándares TIC desde la dimensión pedagógica: Una perspectiva desde los niveles de apropiación de las TIC en la 
práctica educativa docente. Cali, Colombia: Pontificia Universidad Javeriana.

Vásquez-Aguilar, D., Dominguez, L., \& Mata-Muñoz, R. (2017). Interaprendizaje práctico basado en métodos y competencias para optimizar el conocimiento. Polo de Conocimiento, 2(5), 3-16, e-ISSN: 2550 - 682X. Recuperado de: https://doi.org/10.23857/pc.v2i5.61

Venegas, J. (2017a,b). Valoración del uso de recursos digitales como apoyo a la enseñanza-aprendizaje de las matemáticas en Educación Primaria. Tesis Doctoral. Salamanca, España: Universidad de Salamanca. Recuperado de:

https://doi.org/10.14201/gredos.137426

Villanueva, C., Elizondo, J., Vega, A., \& Gómez, M. (2015). Entornos Personales de Aprendizaje: un sistema centrado en el alumno de la educación superior. Revista de Investigación Educativa de la Escuela de Graduados en Educación, 5(19), 63-71, ISSN: 2007-3003. México: Instituto Tecnológico y de Estudios Superiores de Monterrey. 


\author{
Jenny Patricia León Amendaño \\ e-mail: paty gilary94@hotmail.com
}

Nacida en Cuenca, Ecuador, el 29 de mayo del año 1994. Licenciada en Ciencias de la Educación con mención pedagogía, me gradué en el año 2018 en la Universidad Politécnica Salesiana (UPS), Ecuador; actualmente trabajo como docente en educación general básica en la Unidad Educativa Particular "Santa Mariana de Jesús"; he dado cursos de computación a estudiantes de escuelas fiscales que carecían de profesor de computación; antes de obtener mi licenciatura, tuve un largo trayecto en otras áreas del comercio y administración; posteriormente trabajo en mi campo como profesora. 


\section{Pablo Fernando Cisneros Quintanilla \\ e-mail: pcisneros@ucacue.edu.ec}

Nacido en Cuenca, Ecuador, el 29 de marzo del año

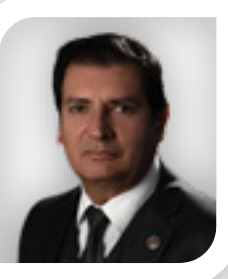
1966. Graduado como Ingeniero Químico en el año 1995 por la Universidad Católica de Cuenca (UCACUE); Especialista en Docencia Universitaria desde el año 2004, por la misma universidad; Magíster en Procesos Educativos Mediados por Tecnología en el 2015, por la Universidad Nacional de Córdoba (UNC), Argentina; y Doctor en Ciencias Pedagógicas, en el 2018, en la Universidad de La Habana (UH), Cuba; Jefe de Otras Modalidades de Estudio en la Universidad Católica de Cuenca (UCACUE), Ecuador, en la que ha ejercido la docencia por 32 años; Profesor de Postgrado en las Universidad Católica de Cuenca y la Universidad Técnica de Manabí (UTM), Ecuador; y Profesor extranjero invitado de la Maestría en Educación, con categoría de Programa de Excelencia del Instituto Pedagógico Latinoamericano y Caribeño (IPLAC), La Habana, Cuba.

El contenido de este manuscrito se difunde bajo una Licencia de Creative Commons ReconocimientoNoComercial-Compartirlgual 4.0 Internacional 